\title{
Seleção de material para ponteiras de bengalas de deficientes visuais com apoio do método TOPSIS
}

\author{
Cláudia Canto de Souza Leão ${ }^{{ }^{*}}$ \\ Alessandro de Castro Corrêa ${ }^{1}$ \\ Jean da Silva Rodrigues ${ }^{1}$ \\ Elza Monteiro Leão Filha ${ }^{2}$
}

\begin{abstract}
Resumo
Este artigo tem por objetivo selecionar materiais aplicáveis à construção de ponteiras de baixo custo para bengalas desmontáveis de deficientes visuais, considerando múltiplos atributos. Foram avaliados cinco ponteiras: uma confeccionada em plástico ABS (Acrilonitrila Butadieno Estireno) por prototipagem de impressão 3D, e outras quatro em compósito de matriz polimérica com incorporação de resíduo particulado de borracha de pneus inservíveis, variando-se o tipo de matriz, epóxi ou poliéster, e com a fração mássica de partículas em dez e quinze por cento. Incluíram-se avaliações de usuários e uma medida de contribuição ambiental. O estudo envolveu três etapas: a experimental em laboratório; experimental em campo; e a avaliação multiatributo, utilizando Technique for Order Preference by Similarity to Ideal Solution (TOPSIS). Os pesos dos atributos foram determinados com base em uma ordenação de importância fornecida por um especialista. Os resultados revelaram que a ponteira de matriz epóxi com partículas em quinze por cento de borracha de pneu apresentou o melhor desempenho.
\end{abstract}

Palavras-chave: Ponteiras; Compósitos; TOPSIS.

\section{Material selection of walking stick tips for visually impaired people using TOPSIS method}

\begin{abstract}
The objective of this study was to select materials for low-cost walking stick tips manufactured for visually impaired people, considering multiple attributes. Five tips were evaluated: one composed of acrylonitrile butadiene styrene (ABS) by $3 \mathrm{D}$ printing and four polymer matrix composites, epoxy or polyester, containing five or fifteen percent particulate rubber from wasted automobile tires. User assessments and an environmental contribution measure were added to the technical attributes. The study was carried out in three stages: laboratory experiments, field experiments, and multi-attribute evaluation using Technique for Order Preference by Similarity to Ideal Solution (TOPSIS). The attributes weights were determined based on a complete rank-ordering provided by expert engineering. The results obtained show that epoxy matrix tips containing fifteen percent of particulate rubber were the best alternative.
\end{abstract}

Keywords: Walking stick tips; Composites; TOPSIS.

\section{Introdução}

Um dos grandes inconvenientes experimentados por pessoas com deficiência visual na utilização de bengalas brancas, principalmente as de baixa renda, é a substituição das ponteiras que se desgastam rapidamente, prejudicando a funcionalidade do equipamento e a qualidade de vida dos usuários.
Ao transitar por vias públicas em geral, os deficientes visuais ficam expostos a constantes situações de riscos e, normalmente, não têm acesso às informações necessárias para sua orientação e locomoção. A simples tarefa de mobilidade e orientação se torna um desafio, uma vez

'Programa de Pós-graduação em Engenharia de Materiais, Instituto Federal de Educação, Ciência e Tecnologia do Pará - IFPA, Belém, PA, Brasil. ${ }^{2}$ Programa de Pós-graduação em Engenharia Industrial, Universidade Federal do Pará - UFPA, Belém, PA, Brasil.

*Autor correspondente: claudialeaoep@hotmail.com

2176-1523 (C) 2020. Leão et al. Publicado pela ABM. Este é um artigo publicado em acesso aberto (Open Access) sob a licença Creative Commons Attribution, que permite uso, distribuição e reprodução em qualquer meio, sem restrições desde que o trabalho original seja corretamente citado. 
que tais ações exigem bom processo cognitivo que inclui a percepção, aprendizagem e memória das informações do ambiente. As principais dificuldades encontradas por um deficiente visual durante sua locomoção independente podem ser minimizadas com o uso de uma bengala mais adaptada, melhorando a sua qualidade de vida. Assim, pode ser possível identificar objetos ou obstáculos no percurso, ou nas imediações, de forma mais eficiente, evitando possíveis colisões e aumentando a agilidade durante a locomoção [1].

A bengala é um instrumento básico de locomoção que garante à pessoa com deficiência visual o direito de ir e vir com segurança ao caminhar, executar tarefas com autonomia e preservar a sua privacidade, por possibilitar deslocamento sem a necessidade de um acompanhante [2]. Trata-se de um instrumento que diminui o impacto da deficiência, auxiliando na independência motora, funcionando como "olhos do cego", servindo para que a pessoa "perceba" os obstáculos que estão à sua volta [3].

Existem muitos modelos de bengalas, entre elas a mais comum é a dobrável. A bengala branca ou desmontável, ilustrada na Figura 1, foi introduzida no final da década de 40 e continua a ser o instrumento mais utilizado na locomoção dos cegos, devido o seu baixo custo, leveza e portabilidade, fácil utilização e uma razoável sondagem do espaço e superfície frontal. A maioria é fabricada em liga de alumínio estrutural, série A356, com comprimentos que variam entre $1,12 \mathrm{~m}$ e $1,37 \mathrm{~m}$. Todavia, a ponteira da bengala apresenta normalmente baixa resistência e pouca durabilidade devido à forma de utilização e a frequência de uso [3].

A Figura 1 exibe a imagem de uma bengala desdobrável para deficientes visuais com a identificação de cada elemento.

Os materiais constituintes compõem outro aspecto importante para o uso de bengalas. A ponteira é uma das partes mais vulneráveis das bengalas desmontáveis. Sua durabilidade varia de acordo com a usabilidade, mas existem casos em que o tempo útil varia entre três a seis meses. As bengalas apresentam as ponteiras fabricadas em diversos materiais, entre os principais estão os polímeros termoplásticos.

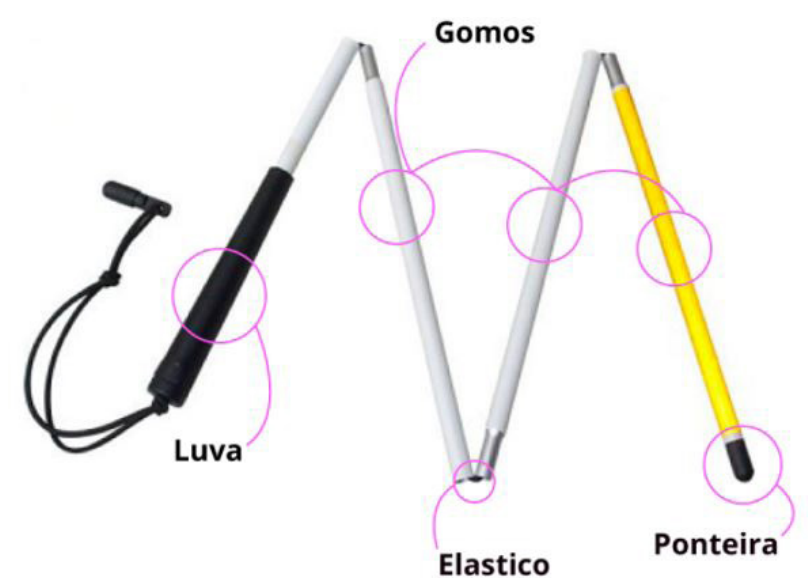

Figura 1. Bengala branca desdobrável.
A ausência da ponteira prejudica a funcionalidade da bengala tanto em termos de aderência quanto em termos de propagação de sons indispensáveis à percepção do usuário. Assim, a Associação de Cegos e Para Cegos do Pará solicitou ao Curso de Mecânica do Instituto Federal do Pará o desenvolvimento de ponteiras capazes de substituir as disponíveis no mercado por terem observado que o alto preço das ponteiras e sua indisponibilidade na Região Metropolitana de Belém constituem um entrave à locomoção independente dos deficientes visuais de baixo poder aquisitivo.

São avaliados dois tipos diferentes de ponteiras, de materiais poliméricos, uma construída em plástico ABS (Acrilonitrila Butadieno Estireno) por prototipagem de impressão 3D, e outra fabricada em compósito de matriz polimérica com incorporação de resíduo particulado de borracha de pneus inservíveis, variando-se o tipo de matriz, epóxi ou poliéster, e com a fração mássica de partículas em $10 \%$ e $15 \%$.

A prototipagem 3D é uma técnica de construção que não exige a modelagem prévia nem a construção de moldes para a concepção de uma peça, o que simplifica o desenvolvimento e a manufatura de um produto, reduzindo o tempo e os custos envolvidos.

Busca-se contribuir também para área de materiais compósitos constituídos com partículas, experimentando a possibilidade de utilização de partículas de borracha oriundas de pneus inservíveis como componente para a ponteira de bengalas de deficientes visuais.

No Brasil, uma parte dos pneus inservíveis é reaproveitada de diversas formas, depois de ser moída e separada dos demais componentes do pneu, especialmente do aço, que também é reutilizado. Entre os produtos que reutilizam a borracha estão solados de sapato, materiais de vedação, dutos pluviais, pisos para quadras poliesportivas, pisos industriais e tapetes para automóveis. A borracha moída e separada também é misturada ao asfalto para uso em pavimentação, gerando o asfalto borracha, que apresenta importantes vantagens. A maioria é, no entanto, queimada como combustível alternativo nas indústrias de cimento. Todas estas destinações são aprovadas pelo IBAMA como ambientalmente adequadas [4].

Segundo Marinucci [5], um compósito é um material que possui dois ou mais constituintes quimicamente diferentes, apresentando em escala macroscópica uma interface bem definida separando esses elementos que em conjunto, originam propriedades diferentes das de seus elementos tomados separadamente. As propriedades dos compósitos estão diretamente ligadas às propriedades dos elementos constituintes do material, assim como a concentração ou fração volumétrica das fases, interface e adesão, disposição das camadas e orientação, bem como pela geometria da partícula.

Há experimentações de compósitos com resíduo de borracha de pneus em diversas matrizes. Como exemplo, Rodrigues e Ferreira [6] utiliza a borracha em uma aplicação em elementos construtivos, como recobrimento de superfícies, onde se faz necessária a absorção e o amortecimento 
de energia(impacto), além da atenuação de ruídos. Para Regis [7] a borracha é também avaliada por seu controle de vibração. Ela é extensivamente usada em acoplamentos flexíveis. Todas as borrachas podem ser usadas para controle de energia e podem ser compostas para variar sua resiliência fundamental para os requerimentos exatos do projetista.

De acordo com essas aplicações, a borracha do pneu foi estrategicamente incorporada ao material da ponteira visando aproveitar algumas de suas boas características mecânicas como a absorção e o amortecimento de energia (impacto),contribuindo com o fator econômico, pois entre os materiais que tem menor custo estão os materiais reciclados, uma vez que são obtidos a partir de rejeitos industriais, além da contribuição ambiental com a reutilização de resíduo inservíveis.

A resina epóxi é um polímero termofixo altamente versátil devido a sua combinação singular de propriedades, como resistência à tração e tenacidade, resistência química, à umidade e à corrosão, boas propriedades térmicas, adesivas $\mathrm{e}$ elétricas. Essas características normalmente não são encontradas em outros materiais poliméricos, sendo sua aplicação atrativa quando se deseja produzir materiais de engenharia.

Para a seleção do material, pode ser necessário avaliar simultaneamente atributos quantitativos e qualitativos, dos quais alguns são objetivos e facilmente mensuráveis, outros, por outro lado, são de natureza subjetiva, mais difíceis de mensurar, porém que vêm progressivamente assumindo maior importância na escolha de materiais.

Nesse caso, os métodos multiatributos são ferramentas valiosas que podem auxiliar o engenheiro de materiais em sua busca das melhores soluções para as aplicações num ambiente de alta tecnologia em que precisa atender a atributos técnicos, ambientais, mercadológicos e sociais $[8,9]$.

O objetivo deste trabalho é a seleção de materiais aplicáveis à construção de ponteiras de baixo custo para bengalas desmontáveis de deficientes visuais, considerando atributos técnicos, custos, conforto, manuseabilidade e contribuição ambienta, é realizada com o apoio do Método TOPSIS, visando a solução dos problemas enfrentados pelos deficientes na Região Metropolitana de Belém

\section{Método de seleção TOPSIS}

Os métodos multiatributos (Multi-atributte Decison Methods - MADM) podem ser definidos como um conjunto de técnicas destinadas a apoiar a análise de problemas complexos que contenham um número limitado e discreto de alternativas, avaliadas com base em diversos atributos cujos níveis de importância sejam variados ou mesmo conflitantes. Os atributos são as características que determinam o desempenho de cada alternativa em relação à aplicação a que se destinam, aqueles que se deseja maximizar são geralmente denominados benefícios e os que se deseja minimizar são genericamente denominados custos ou não benefícios [8-11].
A Technique for Order Preference by Similarity to Ideal Solution (TOPSIS), desenvolvido por Hwang e Yoon [10], é um MADM segundo a qual a melhor alternativa é aquele que mais se aproxima da solução ideal e que mais se afasta da solução anti-ideal. A solução ideal é aquela que maximiza os benefíciose minimiza os custos, ao passo que a anti-ideal maximiza os custos e minimiza os benefícios. Logo, a solução ideal positiva é composta de todos os melhores valores atingíveis dos critérios de benefício; já a solução ideal negativa consiste em todos os piores valores atingíveis dos critérios de custo $[11,12]$.

A simplicidade dos cálculos do TOPSIS é um dos seus atrativos, pois não exige a utilização ou licença de um software de difícil acesso, podendo inclusive ser desenvolvidos em planilha eletrônica. As suas etapas são as seguintes:

1. As medidas de desempenho importante para aplicação devem ser definidas e coletadas das $m$ alternativas, organizando-as numa matriz de decisão conforme Equação 1:

$$
X=\left[x_{i j}\right]_{m \times n} ; i=1, \ldots, m ; j=1, \ldots, n
$$

Em que $x_{\mathrm{ij}}$ representam ao desempenho da alternativa $\mathrm{i}$ associado ao atributoj.

2. Calculam-se os valores normalizados $\left(r_{\mathrm{ij}}\right)$ de cada medida de desempenho $\left(x_{\mathrm{ij}}\right)$ por meio da Equação 2

$$
r_{i j}=\frac{x_{i j}}{\sqrt{\sum_{i=1}^{m} x_{i j}^{2}}} ; i=1, \ldots, m ; j=1, \ldots, n
$$

3. Sendo a importância de cada atributo $j$ descrito por um peso $\left(w_{\mathrm{j}}\right)$.

O TOPSIS não especifica o método de determinação dos pesos, ficando a critério do analista a adoção aquele que julgar mais adequado. Diante de eventuais dificuldades de atribuição dos valores aos pesos, pode-se aplicar o método da somatória de postos (rank sum weights) que recorre à informação da ordenação de atributos [9]. Por esse método, o decisor ou especialista organiza os atributos em ordem decrescente de importância sendo 1, o mais importante, seguindo a estimação dos pesos pela Equação 3.

$$
w_{j}=\frac{\left(n-p_{j}+l\right)}{\sum_{j=1}^{n}\left(n-p_{j}+l\right)}
$$

em que $n$ é o total de atributos e $p$ representa a posição do atributo $j$ na ordenação.

4. Calculam-se os valores normalizados e ponderados de desempenho $\left(v_{\mathrm{j}}\right)$ com a Equação 4. 


$$
v_{i j}=w_{j} r_{i j} ; \sum_{j=1}^{n} w_{j}=1 ; i=1, \ldots, m ; j=1, \ldots, n
$$

5. Identificam-se as soluções ideais $\left(A^{+}\right)$e anti-ideais $\left(A^{-}\right)$através das Equações 5 e 6:

$$
\begin{aligned}
& A^{+}=\left\{v_{1}^{+}, v_{2}^{+}, \ldots, v_{j}^{+}, \ldots v_{n}^{+}\right\}=\left\{\left(\max _{i} v_{i j} \mid j \in J_{I}\right),\right. \\
& \left.\left(\min _{i} v_{i j} \mid j \in J_{2}\right) \mid i=1, \ldots, m\right\}
\end{aligned}
$$

$$
\begin{aligned}
& A^{-}=\left\{v_{1}^{-}, v_{2}^{-}, \ldots, v_{j}^{-}, \ldots v_{n}^{-}\right\}=\left\{\left(\min _{i} v_{i j} \mid j \in J_{1}\right),\right. \\
& \left.\left(\max _{i} v_{i j} \mid j \in J_{2}\right) \mid i=1, \ldots, m\right\}
\end{aligned}
$$

Nas quais $J_{1}$ é o conjunto de benefícios e $J_{2}$ é o conjunto de atributos de custos ou resultados que se pretende minimizar.

6. As distâncias de cada alternativa das soluções ideais $\left(d_{i}^{+}\right)$e das anti-ideais $\left(d_{i}^{-}\right)$são calculadas, utilizando-se a distância Euclidiana, conforme Equações 7 e 8:

$$
\begin{aligned}
& d_{i}^{+}=\sqrt{\sum_{j=1}^{n}\left(v_{i j}-v_{i}^{+}\right)^{2}} ; i=1, \ldots, m \\
& d_{i}^{-}=\sqrt{\sum_{j=1}^{n}\left(v_{i j}-v_{i}^{-}\right)^{2}} ; i=1, \ldots, m
\end{aligned}
$$

7. Por fim, calcula-se a medida de proximidade $\left(C_{i}\right)$ de cada alternativa, permitindo a ordenação das alternativas, sendo a com maior pontuação a avaliada como a melhor (Equação 9).

$$
C_{i}=\frac{d_{i}^{-}}{d_{i}^{+}+d_{i}^{-}}
$$

\section{Materiais e métodos}

Foram avaliadas cinco ponteiras, uma confeccionada em plástico comercial Acrilonitrila Butadieno Estireno (ABS) por prototipagem em impressora $3 \mathrm{D}$, duas em compósitos de matriz polimérica epóxi ARALDITE GY 250, não modificada baseada em Bisfenol, com incorporação de resíduo particulado de borracha de pneus inservíveis em 10\% e 15\% de fração mássica, doravante E10 e E15, e dois compósitos de matriz polimérica de poliéster insaturado ortoftálico de baixa reatividade, cristal, fabricado pela Embrapol sobre denominação comercial ALPHA 190, com 10\% e 15\% de fração mássica de resíduo particulado de borracha de pneus em sua composição, doravante P10 e P15.

Os compósitos foram produzidos por moldagem em molde metálico bipartido, sem pressão. O resíduo de borracha foi inicialmente peneirado em peneira de abertura de $0,35 \mathrm{~mm}$, coletando-se o material passante para a incorporação na matriz de cada compósito, determinando-se a massa para cada fração estabelecida. Adicionado o resíduo à matriz, a esta foi misturado o agente de cura, peróxido de MEK, na proporção de $1 \%$ (v/v) e $0,5 \%$ (v/v) de acelerador de cobalto, no caso da matriz de poliéster; e endurecedor ARADUR, em proporção de $50 \%(\mathrm{v} / \mathrm{v})$ para a matriz de epóxi. Depois de homogeneizadas por 1 minuto, as misturas foram então vertidas nos moldes e curadas à temperatura ambiente por $24 \mathrm{~h}$.

As ponteiras em ABS foram produzidas em uma impressora 3D marca GTMax 3D Core A1V2 com injetor de 0,4 mm, com densidade de enchimento de $99 \%$, com suporte e altura de camada de $0,2 \mathrm{~mm}$.

A Figura 2 ilustra parte das ponteiras produzidas: em (a) mostra-se a ponteira em compósito de matriz epóxi com $10 \%$, em massa, de resíduo de borracha; em (b) é ilustrada a ponteira produzida em ABS pelo uso de impressora 3D.

O procedimento metodológico de seleção foi realizado em três etapas: (i) a experimental, em laboratório, que envolveu a caracterização dos materiais, para a obtenção das variáveis técnicas, através de ensaios mecânicos e físicos; (ii) experimental em campo, por meio de uma pesquisa com os deficientes visuais de uma associação local para a obtenção das variáveis qualitativas; e (iii) a avaliação multiatributo, na qual foram calculados as pontuações globais de desempenho dos materiais das ponteiras avaliadas.

$\mathrm{Na}$ fase experimental nos laboratórios, foi realizada a fabricação dos corpos de provas para as caracterizações dos materiais (borracha, resinas, compósitos e ABS) através dos ensaios e a produção da própria ponteira para a futura fase experimental em campo, para os testes dos usuários.

Para a caracterização física dos materiais, foram realizados ensaios de determinação de massa específica e

(a)

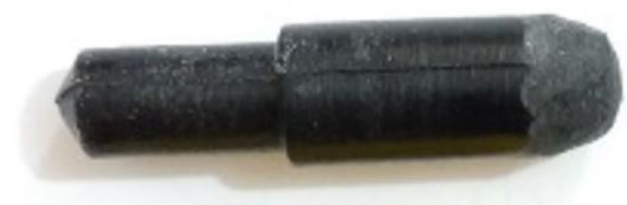

(b)

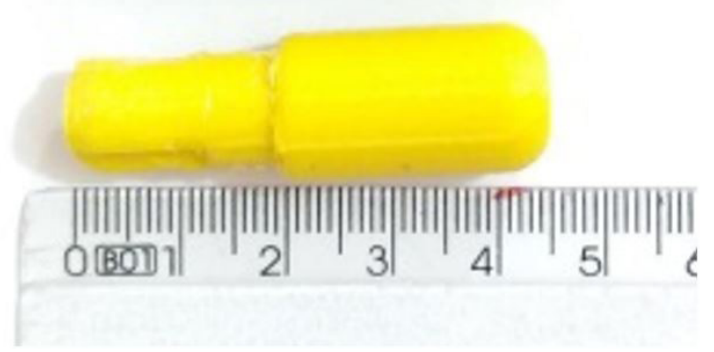

Figura 2. Ponteiras em compósito de matriz epóxi e 10\%, em massa, de borracha de pneus (a) e construída em ABS por impressora 3D (b). 
porosidade, segundo procedimento recomendado em Levy e Pardini [12], e absorção de umidade, conforme ASTM D570 [13]. Para a caracterização mecânica, foram realizados ensaios de tração, conforme ASTM D638 [14], em máquina universal de ensaio AROTEC WDW-1000E com velocidade de $5 \mathrm{~mm} / \mathrm{min}$, e de impacto Charpy, em conformidade com a norma ASTM D256 [15], em equipamento WP 410 da Gunt Hamburg, com martelo de $300 \mathrm{Nm}$.

$\mathrm{Na}$ fase experimental em campo, foi realizada uma entrevista com os usuários deficientes visuais para a identificação dos resultados de satisfação após o teste de usabilidade das ponteiras de cada material desenvolvido. Os usuários foram solicitados a ordenarem as ponteiras em termos de satisfação, sendo a 1 a preferível, no tocante à propagação do som resistência ao atrito e manuseabilidade.

A fase da avaliação multiatributo consistiu no tratamento das informações em conjunto para a seleção do material mais adequado para ponteiras de bengalas. A Tabela 1 apresenta os atributos considerados na análise.

A resistência atração, resistência a impacto, absorção de umidade e massa específica compõem as medidas técnicas de avaliação da caracterização do material. O custo da ponteira foi considerado a depreciação dos equipamentos, energia despendida, mão-de-obra e a matéria prima utilizadas, todos os cálculos estão disponíveis em planilha eletrônica através de solicitações aos autores por correio eletrônico. A Tabela 2 apresenta os custos de cada ponteira calculada e o custo médio da ponteira comercial pesquisada em internet.

Os atributos qualitativos relativos à utilização (propagações do som, resistência ao atrito, manuseabilidade) foram baseadas na percepção de quatro usuários e mensuradas através da escala comparativa "somatória dos postos". A coleta de dados foi realizada por meio de um questionário para a obtenção da pontuação individual após o período do teste de usabilidade por parte dos usuários deficientes que durou três semanas com a ponteira de cada material.

A contribuição ambiental dos materiais foi estimada por meio do método de Saaty [16], segundo o qual os materiais são organizados numa matriz $\left[a_{i j}\right]$ e cada material na linha da matriz é comparado com o material da coluna, formando quocientes de comparações $a_{i j}=s_{i} / s_{j}$ em que $s_{i}$ é a preferência pelo material na linha e $s_{j}$, preferência pelo material na coluna. O julgamento é realizado com o apoio de uma escala de intensidade (Tabela 3 ).
As pontuações de contribuição ambiental (CA) são os elementos do vetor normalizado das médias geométricas das linhas de $\left[a_{i j}\right]$, conforme demonstrado na Tabela 4, na qual se observa que, os materiais que aproveitam resíduos de borracha em 15\% de sua composição (P15 e E15) foram considerados extremamente mais importante do que o ABS. Os que aproveitaram 10\% (P10 e E10), considerados entre muito e extremamente mais importante do que o ABS.

Depois de estruturado o conjunto de atributos do processo de avaliação foi necessário definir as importâncias relativas ou pesos em relação ao objetivo almejado, ou seja, a priorização das características principais da ponteira. Tendo em vista a dificuldade de se obter valores, utilizou-se o método da somatória de postos (rank sum weights) que estima os pesos a partir da informação da ordenação dos atributos. A ordenação dos atributos foi determinada a partir da avaliação de um engenheiro especialista na área de materiais e processos, considerando a aplicação a que se destina o projeto.

\section{Resultados e discussões}

Os resultados da caracterização mecânica dos materiais, Resistência a Tração (RT) e Resistência a Impacto (RI), e física, Absorção de umidade (AU) e Massa específica (ME) são apresentados na Tabela 5. Os dados mostram que as ponteiras fabricadas em $\mathrm{ABS}$ obtiveram melhor desempenho mecânico, tanto em tração quanto em impacto. Em relação a absorção de água e massa específica, as ponteiras de ABS e aquelas fabricadas em compósito de matriz epóxi com $15 \%$ de incorporação de borracha se destacaram, respectivamente, pela baixa absorção de água e mais baixa massa específica dentre as alternativas pesquisadas.

Tabela 2. Custos das ponteiras estudadas em comparação com modelo comercial

\begin{tabular}{cc}
\hline Ponteira & Custo (R\$) \\
\hline P10 & 0,19 \\
P15 & 0,19 \\
E10 & 0,28 \\
E15 & 0,28 \\
ABS & 1,64 \\
COMERCIAL & 8,50 \\
\hline
\end{tabular}

Tabela 1. Atributos utilizados na avaliação

\begin{tabular}{lccccc}
\hline $\mathbf{i}$ & Atributos & Código & Tipo & Direção & Unidade \\
\hline 1 & Resistência a tração & RT & Quantitativo & Max & MPa \\
2 & Resistência a impacto & RI & Quantitativo & Max & MPa \\
3 & Absorção de umidade & AU & Quantitativo & Min & Proporção \\
4 & Massa específica & ME & Quantitativo & Max & Proporção \\
5 & Custo & C & Quantitativo & Min & R\$ \\
6 & Percepção da propagação do som & PS & Qualitativo & Max & Pontos \\
7 & Percepção da resistência ao atrito & RA & Qualitativo & Max & Pontos \\
8 & Manuseabilidade & MP & Qualitativo & Max & Pontos \\
9 & Contribuição ambiental & CA & Qualitativo & Max & Saaty \\
\hline
\end{tabular}


A Tabela 5 traz ainda os valores do Custo (C), Percepção da propagação do som (PS), Percepção da resistência ao atrito (RA), Manuseabilidade (MP) e Contribuição Ambiental (CA), compondo assim a matriz de decisão com a identificação do objetivo desejado para cada atributo.

Pode-se observar a presença de características típicas de um problema multiatributo, justificando a opção pelos métodos MADM para a seleção do material da ponteira. Primeiramente, o número limitado de alternativas à disposição (no caso, cinco tipos de ponteiras). Adicionalmente, são utilizadas, na avaliação, medidas quantitativas, como Resistência à tração, e medidas qualitativas, como a percepção da propagação do som, as quais foram estimadas subjetivamente pelos usuários por meio de uma escala. Outra característica é a relação conflituosa entre atributos, como sugere o comportamento entre $\mathrm{C}$ em relação à RT e a RI. Por fim, a necessidade de que o decisor explicite a preferência por cada um dos atributos para melhor adequação à aplicação a que se destina o material.

A Tabela 6 apresenta os pesos dos atributos calculados por meio da Equação 3, com base na ordenação obtida a partir de uma entrevista com um profissional técnico da área, solicitando quais os critérios mais importantes no processo de avaliação da ponteira da bengala.

A seguir, na Tabela 7, são apresentados os resultados referentes aos cálculos da matriz com dados normalizados, por meio da Equação 2, e, na Tabela 8, os dados normalizados e ponderados de acordo com os pesos estipulados, conforme Equação 4.

A Tabela 9 apresenta os valores das soluções ideais e anti-ideais conforme o objetivo de cada critério da matriz decisão, como definidos nas Equações 5 e 6.

Por fim, as pontuações globais de desempenho de cada material ou medidas de proximidade calculadas pelo TOPSIS, conforme Equação 9 são apresentadas na Tabela 10, revelando que a ponteira de matriz Epoxi com $15 \%$ de partículas de borracha apresentou um desempenho superior, com a maior pontuação $\left(\mathrm{C}_{\mathrm{i}}=0,664\right)$ em relação às outras ponteira sendo seguida da ponteira de matriz Epoxi com $10 \%$ de partículas de borracha.

Todos os cálculos, em planilha eletrônica e em linguagem R, estão disponíveis através de solicitações por correio eletrônico aos autores.

Tabela 3. Escala de intensidade de preferências de Saaty [16]

\begin{tabular}{ccccc}
\hline Igual & $\begin{array}{c}\text { Moderadamente mais } \\
\text { importante }\end{array}$ & Fortemente mais importante & Muito mais importante & $\begin{array}{c}\text { Extremamente mais } \\
\text { importante }\end{array}$ \\
\hline 1 & 3 & 5 & 7 & 9 \\
\hline
\end{tabular}

Tabela 4. Matriz de comparações e cálculo da contribuição ambiental dos materiais

\begin{tabular}{c|ccccc|c|c}
\hline & P10 & P15 & E10 & E15 & ABS & g & CA \\
\hline P10 & 1 & $1 / 2$ & 1 & $1 / 2$ & 8 & 1,149 & 0,175 \\
P15 & 2 & 1 & 2 & 1 & 9 & 2,048 & 0,312 \\
E10 & 1 & $1 / 2$ & 1 & $1 / 2$ & 8 & 1,149 & 0,175 \\
E15 & 2 & 1 & 2 & 1 & 9 & 2,048 & 0,312 \\
ABS & $1 / 8$ & $1 / 9$ & $1 / 8$ & $1 / 9$ & 1 & 0,181 & 0,027 \\
\hline
\end{tabular}

Nota: P10 = Matriz poliéster com 10\% de borracha; P15 = Matriz poliéster com $15 \%$ de borracha; E10 = Matriz epoxi com 10\% de borracha; E15= Matriz epoxi com 15\% de borracha; $\mathrm{ABS}$ = Acrilonitrila Butadieno Estireno; $\mathrm{g}$ = média geométrica; $\mathrm{CA}$ = pontuação de contribuição ambiental.

Tabela 5. Matriz de decisão multiatributo

\begin{tabular}{cccccccccc}
\hline Material & RT & RI & AU & ME & C & PS & RA & MP & CA \\
\hline Medida & MPa & J/m & $\mathbf{~} \mathbf{m}^{2}$ & $\mathbf{g} / \mathbf{c m}^{3}$ & R\$/unid & Pts & Pts & pts & pts \\
\hline Obj. & Max. & Max. & Min. & Max. & Min. & Max & Min. & Max. & Max. \\
\hline P10 & 4,59 & 2,32 & 1,48 & 1,06 & 0,19 & 0,4 & 0,67 & 0,87 & 0,17 \\
P15 & 3,20 & 2,62 & 1,78 & 0,91 & 0,19 & 0,33 & 0,47 & 0,67 & 0,31 \\
E10 & 14,50 & 2,62 & 0,88 & 1,10 & 0,28 & 0,87 & 0,80 & 0,73 & 0,17 \\
E15 & 14,41 & 2,92 & 0,47 & 0,90 & 0,28 & 0,66 & 0,60 & 0,53 & 0,31 \\
ABS & 26,00 & 3,52 & 0,40 & 0,95 & 1,64 & 0,73 & 0,47 & 0,20 & 0,03 \\
\hline TOTAL & 62,70 & 14 & 5,01 & 4,92 & 2,58 & 3,00 & 3,00 & 3,00 & 1,00 \\
\hline
\end{tabular}

Nota: RT = Resistência a Tração; RI = Resistência a impacto; AU = Absorção de umidade; ME = Massa específica; C $=$ Custo; PS $=$ Percepção da propagação do som; RA = Percepção da resistência ao atrito; $\mathrm{MP}=$ Manuseabilidade; $\mathrm{CA}=$ Contribuição ambiental.

Tabela 6. Pesos dos atributos com base em suas ordenações

\begin{tabular}{cccccccccc}
\hline Atributos & RT & RI & AU & ME & C & PS & RA & MP & CA \\
\hline $\mathrm{p}_{\mathrm{j}}$ & 4 & 1 & 7 & 6 & 5 & 2 & 3 & 8 & 4 \\
$\mathrm{n}-\mathrm{p}_{\mathrm{j}}-1$ & 6 & 9 & 3 & 4 & 5 & 8 & 7 & 2 & 1 \\
$\mathrm{w}_{\mathrm{j}}$ & 0,133 & 0,200 & 0,067 & 0,089 & 0,111 & 0,178 & 0,156 & 0,044 & 0,022 \\
\hline
\end{tabular}

Nota: RT = Resistência a Tração; RI = Resistência a impacto; $\mathrm{AU}=$ Absorção de umidade; $\mathrm{ME}=$ Massa específica; $\mathrm{C}=\mathrm{Custo}$; PS = Percepção da propagação do som; RA = Percepção da resistência ao atrito; $\mathrm{MP}=$ Manuseabilidade; $\mathrm{CA}=$ Contribuição ambiental. 
Tabela 7. Matriz com os dados normalizados

\begin{tabular}{ccccccccccc}
\hline Material & RT & RI & AU & ME & C & PS & RA & MP & CA \\
\hline P10 & 0,137 & 0,367 & 0,580 & 0,480 & 0,111 & 0,283 & 0,486 & 0,604 & 0,345 \\
P15 & 0,095 & 0,414 & 0,697 & 0,412 & 0,111 & 0,235 & 0,340 & 0,465 & 0,616 \\
E10 & 0,432 & 0,414 & 0,345 & 0,498 & 0,164 & 0,612 & 0,583 & 0,511 & 0,345 \\
E15 & 0,430 & 0,462 & 0,184 & 0,408 & 0,164 & 0,471 & 0,438 & 0,372 & 0,616 \\
ABS & 0,775 & 0,556 & 0,157 & 0,430 & 0,960 & 0,518 & 0,340 & 0,139 & 0,054 \\
\hline
\end{tabular}

Nota: RT = Resistência a Tração; RI = Resistência a impacto; AU = Absorção de umidade; $\mathrm{ME}=$ Massa específica; $\mathrm{C}=\mathrm{Custo}$; PS = Percepção da propagação do som; $\mathrm{RA}=$ Percepção da resistência ao atrito; $\mathrm{MP}=$ Manuseabilidade; $\mathrm{CA}=$ Contribuição ambiental.

Tabela 8. Dados normalizados e ponderados

\begin{tabular}{ccccccccccc}
\hline Material & RT & RI & AU & ME & C & PS & RA & MP & CA \\
\hline P10 & 0,018 & 0,073 & 0,039 & 0,043 & 0,012 & 0,050 & 0,076 & 0,027 & 0,008 \\
P15 & 0,013 & 0,083 & 0,046 & 0,037 & 0,012 & 0,042 & 0,053 & 0,021 & 0,014 \\
E10 & 0,058 & 0,083 & 0,023 & 0,044 & 0,018 & 0,109 & 0,091 & 0,023 & 0,008 \\
E15 & 0,057 & 0,092 & 0,012 & 0,036 & 0,018 & 0,084 & 0,068 & 0,017 & 0,014 \\
ABS & 0,103 & 0,111 & 0,010 & 0,038 & 0,107 & 0,092 & 0,053 & 0,006 & 0,001 \\
\hline
\end{tabular}

Nota: $\mathrm{RT}=$ Resistência a Tração; RI = Resistência a impacto; AU = Absorção de umidade; ME = Massa específica; C = Custo; PS = Percepção da propagação do som; $\mathrm{RA}=$ Percepção da resistência ao atrito; $\mathrm{MP}=$ Manuseabilidade; $\mathrm{CA}=$ Contribuição ambiental.

Tabela 9. Soluções ideais e anti-ideais

\begin{tabular}{ccccccccccc}
\hline Material & RT & RI & AU & ME & C & PS & RA & MP & CA \\
\hline Objetivo & + & + & - & + & - & + & - & + & + \\
d + & 0,1033 & 0,1113 & 0,0104 & 0,0443 & 0,0124 & 0,1088 & 0,0529 & 0,0269 & 0,0137 \\
$\mathrm{~d}-$ & 0,0127 & 0,0733 & 0,0465 & 0,0362 & 0,1067 & 0,0419 & 0,0908 & 0,0062 & 0,0012 \\
\hline
\end{tabular}

Nota: RT = Resistência a Tração; RI = Resistência a impacto; $\mathrm{AU}=$ Absorção de umidade; $\mathrm{ME}=$ Massa específica $\mathrm{C}=$ Custo; PS $=$ Percepção da propagação do som; RA = Percepção da resistência ao atrito; $\mathrm{MP}=$ Manuseabilidade; $\mathrm{CA}=$ Contribuição ambiental.

Tabela 10. Medidas de proximidade dos processos e ranking final

\begin{tabular}{ccccc}
\hline Material & $\mathbf{d}+$ & $\mathbf{d}-$ & $\mathbf{C}_{\mathbf{i}}$ & RANKING \\
\hline P10 & 0,116 & 0,099 & 0,46 & $4^{\circ}$ \\
P15 & 0,122 & 0,104 & 0,46 & $5^{\circ}$ \\
E10 & 0,068 & 0,124 & 0,647 & $2^{\circ}$ \\
E15 & 0,060 & 0,118 & 0,664 & $1^{\circ}$ \\
ABS & 0,099 & 0,122 & 0,552 & $3^{\text {o }}$ \\
\hline
\end{tabular}

Nota: $d_{i}^{+}=$soluções ideais; $d_{i}^{-}=$soluções anti-ideais; $C_{i}=$ medida de proximidade.

\section{Conclusões}

Os resultados deste trabalho indicaram que as melhores composições de materiais para a ponteira desmontável de deficientes visuais, entre as cinco propostas, foram as de matrizes Epóxis, com 15\% e 10\% de borracha de pneus, respectivamente, primeira e segunda posições.

O método TOPSIS foi considerado satisfatório para o apoio para a análise e demonstrou ser simples e capaz de considerar facilmente um bom número de atributos quantitativos e qualitativos, utilizando recursos computacionais básicos, como planilhas eletrônicas, constituindo-se assim um instrumento de apoio promissor na seleção de materiais.
Na aplicação, foi possível mensurar através da pesquisa de campo com os deficientes, os atributos de satisfação qualitativos, como a propagação do som, a resistência ao atrito e a manutenção da ponteira, aspectos esses que normalmente ficam a margem de uma avaliação, por serem, em decorrência de sua natureza subjetiva, de difícil mensuração.

Importante salientar que o produto em desenvolvimento se destina a usuários de baixa renda e que uma contribuição para o desenvolvimento de produtos de tecnologia assistiva, foi a participação desse público-alvo no processo de avaliação, permitindo uma melhor busca de satisfação de suas necessidades. Pode-se observar que as ponteiras avaliadas exibiram custos inferiores aos cobrados no mercado, sendo uma vantajosa opção aos usuários mais carentes.

O desenvolvimento deste projeto trouxe ainda a iniciativa ambiental, com a reutilização de resíduos de borracha de pneus inservíveis, gerando uma diminuição no custo da matéria prima e colaborando para redução de desse passivo ambiental, encontrando-se uma aplicação para a grande quantidade de borracha descartada provenientes de pneus usados. Com o aproveitamento, foram reduzidos os problemas ambientais e de saúde pública, como proposta de reuso de materiais que seriam descartados em aterros sanitários proliferando doenças. 


\section{Referências}

1 Alves FA, Neumann AM, Gouvêa MM Jr, editor. Bengala inteligente neural baseada em aprendizagem por reforços para deficientes visuais. Belo Horizonte: Laboratório de Robótica e Inteligência Artificial, Pontificia Universidade Católica de Minas Gerais; 2014 [acesso em 8 nov. 2017]. Disponível em: http://www.lbd.dcc.ufmg.br/colecoes/ eniac/2014/0071.pdf

2 Cerqueira JB. Bengala branca: símbolo de independência das pessoas cegas. Rio de Janeiro; 2011 [acesso em 27 jul. 2015]. Disponível em: http://www.bengalalegal.com/bengala-branca

3 Rosa PRM. Bengala de apoio a cegos com deteç̧ão de buracos [dissertação]. Avenco: Universidade de Avenco; 2009.

4 Compromisso Empresarial para Reciclagem. O mercado para reciclagem. São Paulo: CEMPRE; 2019 [acesso em 7 fev. 2019]. Disponível em: http://cempre.org.br/artigo-publicacao/ficha-tecnica/id/7/pneus

5 Marinucci G. Materiais compósitos poliméricos: fundamentos e tecnologia. São Paulo: Artliber; 2011. 333 p.

6 Rodrigues MRP, Ferreira OP. Caracterização de compósitos produzidos com borracha de pneus e resina poliuretana. Revista Iberoamericana de Polímeros. 2010;11(5):274-283.

7 Regis TM. Desenvolvimento e caracterização de compósitos híbridos a partir de polipropileno reciclado, resíduo de borracha de pneu e carbonato de cálcio [dissertação]. São Paulo: Universidade Presbiteriana Machenzie; 2011.

8 Jahan A, Edwards KL, Bahraminasab M. Multi-criteria decision analysis for supporting the selection of engineering materials in product design. Oxford: Butterworth-Heinemann; 2016. $252 \mathrm{p}$.

9 Corrêa AC, Peixoto AL, Corrêa DCG. Métodos multi-atributos: aplicação do COPRAS na seleção de filamentos para produção de maquete em impressora 3D. Bomgeam. 2018;5(1):1-9. http://dx.doi.org/10.31419/ISSN.2594942X.v52018i1a11ACC.

10 Hwang CL, Yoon K. Multiple Attribute Decision Making: methods and applications. New York: Springer-Verlag; 1981. 259 p. http://dx.doi.org/10.1007/978-3-642-48318-9.

11 Gomes LF, Gomes CFS. Tomada de decisão gerencial: um enfoque multicritério. 5. ed. São Paulo: Atlas; 2014.324 p.

12 Levy F No, Pardini LC. Compósitos estruturais: ciência e tecnologia. São Paulo: Edgar Blucher; 2006.

13 American Society for Testing and Materials. ASTM D570: standard test method for water absorption of plastics. West Conshohocken: ASTM; 2010.

14 American Society for Testing and Materials. ASTM D638: standart test method for tensile properties of plastics. West Conshohocken: ASTM; 2010.

15 American Society for Testing and Materials. ASTM D256-4: standard test method for determining the izod pendulum impact resistence of plastics. West Conshohocken: ASTM; 2008.

16 Saaty TL. The analytic hierarchy process: planning, priority, setting and resource allocation. New York: McGrawHill; 1980. 287 p.

Recebido em: 20 Fev. 2019

Aceito em: 20 Jan. 2020 